\title{
TECHNICAL
}

\section{COMMENT Addressing a critique of the TEASI framework for invasive species risk assessment}

Brian Leung, ${ }^{1,2 *}$ Nuria

Roura-Pascual, ${ }^{3,4}$ Sven Bacher, ${ }^{5}$

Jaakko Heikkilä, ${ }^{6}$ Lluis Brotons, ${ }^{4}$

Mark A Burgman, ${ }^{7}$ Katharina

Dehnen-Schmutz, ${ }^{8}$ Franz Essl, ${ }^{9}$

Philip E Hulme, ${ }^{10}$ David M

Richardson, ${ }^{11}$ Daniel Sol ${ }^{12}$ and

Montserrat Vilà ${ }^{13}$

\begin{abstract}
We address criticism that the Transport, Establishment, Abundance, Spread, Impact (TEASI) framework does not facilitate objective mapping of risk assessment methods nor defines best practice. We explain why TEASI is appropriate for mapping, despite inherent challenges, and how TEASI offers considerations for best practices, rather than suggesting one best practice.
\end{abstract}

\section{Keywords}

Colonisation, exotic, habitat suitability, life history trait, non-indigenous, policy, propagule pressure, risk analysis, species distribution, uncertainty.

Ecology Letters (2013) 16: 1415-e6
Our review of alien species risk assessments (RA) (Leung et al. 2012) aimed to synthesise the diverse approaches applied in this field to establish a logical framework for best practices. We believe the TEASI framework that makes explicit the consideration of Transport, Establishment, Abundance, Spread and Impact aspects of biological invasions helps integrate the main ideas underlying risk assessment and identifies important open questions. Barry (2013) provided a thoughtful review of our study and while he found much to commend in our approach, he indicated two main criticisms: (1) the mapping process in the article was subjective and TEASI does not encapsulate all the reviewed RAs and (2) we are not explicit in defining the best practice. We address each criticism.

First, although quantitative approaches were relatively easy to map onto the TEASI framework, scoring-based approaches were more difficult and more subjective. Importantly, subjective does not mean arbitrary. For instance, mapping RA questions such as 'propagules dispersed by wind' onto the Spread component in TEASI and identifying it as a species trait is arguably logical. However, the rationale for how answers were combined was less clear for scoring approaches. For instance, many simply summed binary yes/no answers across all components, so we agree that they 'would need to be radically redefined' to map onto TEASI as many do not consider model structure. Barry (2013) further notes that the scoring approaches 'are abstract while the TEASI model is process-based and explicit'. This is certainly true but if the 'abstract' risks do not (at least imperfectly) map onto the set of real processes underlying invasions, we question whether they can be predictive. Thus, we argue that scoring-based approaches can and should be considered in the context of a process-based framework, but we acknowledge that this is challenging. We view this difficulty in mapping model structure as a limitation of existing scoring methodology rather than of the process-based TEASI model. We pose the questions: do the scoring model structures make sense in terms of invasion processes? How? If they do not, in the future, should they? Note, we do not deny the value of scoring RAs; they will remain important in addressing biological invasions, given limited time, data and resources.

In addition, Barry (2013) argues that TEASI equations were too highly structured and prescriptive. Although we could have just listed factors thought to be relevant for invasion risk, this would be less valuable. Models are useful, in part, exactly because they are highly structured, presenting a clear picture of how we believe factors relate to one another and to invasion risk, and where we disagree with other models. Alternative formulations are possible, but it would be useful to consider their consequences. For instance, scoring approaches sometimes only sum the vector types in Transport. The consequence is that all vectors types are implicitly mod-
${ }^{1}$ Department of Biology, McGill University, Montreal, Quebec, H3A 1B1, Canada

${ }^{2}$ School of Environment, McGill University, Montreal, Quebec, H3A 2A7, Canada

${ }^{3}$ Departament de Ciències Ambientals, Facultat de Ciències, Universitat de Girona, Campus Montilivi, 17071, Girona, Catalonia, Spain

${ }^{4}$ Centre Tecnològic Forestal de Catalunya, Ctra. St. Llorenç de Morunys km 2, 25280, Solsona, Catalonia, Spain

${ }^{5}$ Departement of Biology, Ecology \& Evolution Unit, University of Fribourg,

Chemin du Musée 10,1700, Fribourg, Switzerland

${ }^{6}$ MTT Economic Research, Latokartanonkaari 9, 00790, Helsinki, Finland

${ }^{7}$ Department of Botany, University of Melbourne, Parkville, Australia

${ }^{8}$ School of Life Sciences, University of Warwick, Wellesbourne, Warwick, CV35 gEF, UK
${ }^{9}$ Department of Conservation Biology, Vegetation and Landscape Ecology, Faculty Centre of Biodiversity, University of Vienna, Rennweg 14, 1030,

Vienna, Austria

${ }^{10}$ The Bio-Protection Research Centre, Lincoln University, Christchurch, New Zealand

${ }^{11}$ Department of Botany and Zoology, Centre for Invasion Biology, Stellenbosch University, Matieland, 7602, South Africa

${ }^{12}$ Centre for Ecological Research and Forestry Applications (CREAF), Consejo Superior de Investigaciones Científicas (CEAB-CSIC), Universitat Autònoma de Barcelona, 08193, Cerdanyola del Valles, Catalonia, Spain

${ }^{13}$ Estación Biológica de Doñana, Consejo Superior de Investigaciones Científicas (EBD-CSIC), Avda. Américo Vespucio s/n E-41092, Sevilla, Spain *Correspondence: E-mail: brian.leung2@mcgill.ca 
elled as having equal transport capability and vector numbers factors explicitly considered in TEASI. We stand by the rationale underlying our formulations and the utility of explicitly considering model components, structure and dependencies, while acknowledging that alternative formulations may be necessary (e.g. due to data limitations).

Second, we emphasise that we proposed a 'framework for best practices' and do not suggest that there is a single best approach for risk assessment. Our view in the TEASI framework is that best practices involve, among other issues, considering the major relevant invasion processes from Transport to Impact, use of ecological theory, and consideration of uncertainty. In some cases, certain invasion steps will not be needed, such as for deliberate introductions where the Transport component can be taken as given; here, adaptation of TEASI would be straightforward. In contrast, many RAs do not address Impact, which ultimately should be assessed. Our aim was not to negate the relevance of previous efforts, but to synthesise and build upon them, and we recognise that modification may be needed for particular systems. Nevertheless, we highlight that most existing models only describe part of the picture and perhaps provide an incomplete view of invasion risk. By explicitly modelling components, structure and dependencies, we provide, for the consideration of managers and researchers, guidance for estimating and combining (most) relevant processes.
Finally, we agree that further research is needed and dedicated much of the manuscript to identifying open questions. We highlighted that there have been a number of ways to combine TEASI components, and that the ramifications have generally remained unexplored. We suggested exploration of the relative importance of subcomponents, derivation of rules for excluding subcomponents, and comparison of performance between quantitative and qualitative models, among others. We identified useful future modifications of TEASI (e.g. analogous models for multiple species or pathway risk assessments). Clearly, much work remains to be done. Together, through discussion and collaboration, we may move towards more rational means of assessing the potential risk of alien species.

\section{REFERENCES}

Barry, S. C. (2013) Best practice in alien species risk assessment: a comment on Leung et al. (2012). Ecol. Lett., DOI: 10.1111/ele.12173.

Leung, B., Roura-Pascual, N., Bacher, S., Heikkilä, J., Brotons, L., Burgman, M. A. et al. (2012) TEASIng apart alien species risk assessments: a framework for best practices. Ecol. Lett., 15, 1475-1493.

Editor, Marcel Rejmanek

Manuscript received 15 July 2013

Manuscript accepted 1 August 2013 